\title{
Opciones de gestión para reducir la cacería ilegal: El caso de la Reserva de Vida Silvestre Amazónica Manuripi, Bolivia
}

\author{
S. Espinoza ${ }^{1, *}$, J. Dresdner², C. Chávez ${ }^{3}$
}

(1) Programa de Magíster en Economía de Recursos Naturales y Medio Ambiente, Universidad de Concepción, 160-C, Concepción, Chile.

(2) Departamento de Economía, Núcleo de Investigación en Economía Ambiental y de Recursos Naturales e Interdisciplinary Center for Aquaculture Research (INCAR), Universidad de Concepción, Casilla 1987, Concepción, Chile.

(3) Departamento de Economía, Núcleo de Investigación en Economía Ambiental y de Recursos Naturales e Interdisciplinary Center for Aquaculture Research (INCAR), Universidad de Concepción, Casilla 1987, Concepción, Chile.

* Autor de correspondencia: S. Espinoza [sophiaespinoza@udec.cl]

> Recibido el 31 de enero de 2013, aceptado el 24 de mayo de 2013

Espinoza, S., Dresdner, J., Chávez, C. (2013). Opciones de gestión para reducir la cacería ilegal: El caso de la Reserva de Vida Silvestre Amazónica Manuripi, Bolivia. Ecosistemas 22(2):97-103. Doi.: 10.7818/ECOS.2013.22-2.14

La cacería ilegal representa una amenaza para la conservación de la vida silvestre. Las motivaciones y efectos que puede tener son variables y aún no han sido ampliamente estudiados. Aplicando un enfoque teórico-conceptual económico analizamos los diferentes factores que pueden motivar la cacería ilegal y discutimos las implicaciones de gestión. Basándonos en el caso de la cacería ilegal que ocurre durante la recolección de castaña (Bertholletia excelsa) en la Reserva de Vida Silvestre Amazónica Manuripi, Bolivia, analizamos los resultados de un extensivo estudio de incumplimiento. En base a la revisión de fuentes primarias y secundarias de información y al marco conceptual referido a modelos económicos de disuasión, identificamos que la probabilidad de detección y sanción, la moralidad, la legitimidad y la influencia social pueden afectar el cumplimiento de la prohibición de caza en la Reserva Manuripi. Adicionalmente, consideramos que el conocimiento de la regulación también puede afectar la decisión de cazar y señalamos evidencia empírica que sugiere que el hábito de consumir carne silvestre y la no-disponibilidad de alternativas a la carne silvestre motivan la cacería ilegal en Manuripi. Bajo este análisis, las recomendaciones para disminuir el riesgo de cacería ilegal durante el periodo de recolección de castaña se enfocan en mejorar los esfuerzos de fiscalización, monitoreo y control por parte de la Autoridad Ambiental, paralelamente a la implementación de procesos de concientización dirigidos a modificar los valores éticos y hábitos culturales entre los recolectores.

Palabras clave: cacería ilegal, cumplimiento, regulación a la caza, factores de disuasión.

Espinoza, S., Dresdner, J., Chávez, C. (2013). Policy options to reduce illegal hunting: a case study in the Manuripi Amazon Wildlife Reserve, Bolivia. Ecosistemas 22(2):97-103. Doi.: 10.7818/ECOS.2013.22-2.14

Illegal hunting is a threat to wildlife conservation. The motivations for poaching and its possible effects are variable and have not been extensively studied. Applying an economic theoretical-conceptual frame, we analyze the different factors that may determine illegal hunting and discuss some policy implications. Based on the illegal hunting experience during the Brazil nut harvesting season in the Manuripi Amazon Wildlife Reserve, Bolivia, we analyzed an extensive study of non-compliance. By reviewing primary and secondary sources of information and the deterrent economic models theory, we identified that the probability of detection and sanction, morality, legitimacy and social influence may affect compliance with the hunting prohibition in the Manuripi Reserve. Additionally, we consider that knowledge of the regulation may also affect the decision to hunt. Finally, we found empirical evidence suggesting that habits of eating wild meat among collectors and non-wild meat availability are factors that affect the decision to hunt illegally in the Manuripi Reserve. Based on our analysis, the recommendations to reduce illegal hunting during the Brazil nut harvesting period focus on strengthening the enforcement, monitoring and control efforts by the Environmental Authority, in addition to awareness processes that can change the ethical values and cultural habits among collectors.

Keywords: illegal hunting, compliance behavior, hunting regulation, deterrence factors.

\section{Introducción}

La cacería ilegal es un problema que afecta la conservación de la vida silvestre en diferentes regiones del mundo. Este tipo de cacería tiene diferentes fines, incluyendo la provisión de carne para alimentación y su comercialización o de animales vivos, generando así un mercado ilegal de carne silvestre y el tráfico de especies. Mientras gran parte de la literatura se enfoca en caracterizar y analizar los efectos de la cacería intensiva desarrollada por actores locales en diferentes zonas de aprovechamiento (Wilkie y Carpenter 1999; Bassett 2005; Nielsen 2006; Schenck et al. 2006; Willcox y
Nambu 2007; Brashares et al. 2011), pocos estudios han analizado las motivaciones y efectos que tiene la cacería ilegal, concentrándose la mayor parte de ellos en la región del África (Skonhoft y Solstad 1996; Loibooki et al. 2002; Eliason 2003; Johannesen 2005; Holmern et al. 2007). La cacería ilegal puede generar diferentes niveles de presión sobre las especies, dependiendo de la vulnerabilidad que éstas tengan y de la intensidad de cacería. Bassett (2005) analiza indicadores que evidencian la desaparición de diferentes especies en Costa de Marfil donde si bien la cacería es permitida bajo permisos especiales, ésta es muy abundante e intensiva. 
En cuanto a los factores que motivan la cacería ilegal, algunos estudios encuentran evidencia empírica que relaciona los niveles de riqueza y fuentes de ingresos económicos con la práctica intensiva de la caza (Loibooki et al. 2002; Nielsen 2006; Brashares et al. 2011). Loibooki et al. (2002) encuentran, además, que la accesibilidad a fuentes alternativas de carne reduce la probabilidad de participar en cacería ilegal. Por su parte, Holmern et al. (2007) sugieren que la probabilidad de ser arrestado puede afectar las prácticas ilegales de cacería, pero que ésta varía estacionalmente y también es afectada por el tamaño del grupo de cazadores. Eliason (2003), se basa en la teoría de la Neutralización propuesta por Sykes y Matza (1957) desde la sociología, en la cual se señala que los infractores utilizan diferentes técnicas antes y después de cometer algún acto delictivo, como formas de motivarse, justificarse y evitar un sentimiento de culpa. El autor encuentra que las técnicas de neutralización empleadas por individuos que cazan ilegalmente incluyen: i) la negación de responsabilidad, ii) señalar derecho a beneficiarse de la cacería, iii) considerar que la ley es injusta, y iv) señalar que necesitan de la cacería para abastecerse de carne para alimentación. Adicionalmente, Eliason (2003) encuentra que algunos individuos cazan ilegalmente por la sensación excitante que les produce el participar en una actividad ilegal.

Skonhoft y Solstad (1996) señalan diferencias entre las motivaciones para la cacería ilegal con fines de subsistencia y la cacería ilegal con fines comerciales. Estos autores indican que, además de factores históricos, sociales y culturales, existen incentivos económicos asociados a los costos de oportunidad que tienen los cazadores. En el estudio también se reconoce el efecto que tienen los mayores esfuerzos de control y multas elevadas en la reducción de la cacería ilegal. Schenck et al. (2006), si bien no se enfocan en la cacería ilegal, señalan que la demanda por la carne silvestre, el precio en los diferentes mercados para este tipo de carne o factores culturales asociados a la familiaridad y costumbre por el consumo de la carne silvestre también afectan la cacería.

La evidencia empírica nos muestra que las motivaciones para cazar ilegalmente pueden ser varias y están en función de las características del caso estudiado (Skonhoft y Solstad 1996; Loibooki et al. 2002; Eliason 2003; Holmern et al. 2007). Sin embargo, son pocos los estudios que basan su análisis en un modelo teórico formal bien definido, lo cual permitiría tener un marco para interpretar los resultados y discutir las políticas apropiadas para enfrentar el problema de la cacería ilegal. La legislación que restringe las prácticas de cacería va desde permisos especiales hasta la prohibición completa de la actividad, incluyendo sanciones para los infractores. Sin embargo, la literatura que considera los esfuerzos de fiscalización es poco abundante y conclusiva en este aspecto. Existen ejemplos relacionados con la gestión de pesquerías, donde se han analizado los efectos de las estrategias de fiscalización bajo el concepto económico de maximización de utilidad, el cual señala que los individuos se comportarían ilegalmente si los beneficios asociados a esa actividad superan a los costos (Furlong 1991; Kuperan y Sutinen 1998; Hatcher et al. 2000; Nielsen y Mathiessen 2003; Hatcher y Gordon 2005; Viteri y Chávez 2007; Eggert y Lokina 2010; Dresdner et al. (datos sin publicar)). Sin embargo no se conoce de literatura específica para casos de cacería que hayan considerado este enfoque económico.

El presente artículo pretende utilizar el enfoque teórico-conceptual económico para integrar los diferentes factores que pueden motivar la cacería ilegal, y analizar los posibles determinantes del incumplimiento a las regulaciones que prohíben la cacería. Además, discute las implicaciones de gestión a partir de los resultados encontrados para un caso de estudio particular de la amazonia boliviana. El trabajo se organiza en cinco secciones. A continuación, revisaremos brevemente la literatura económica sobre cumplimiento. En la tercera sección, presentamos el caso de estudio, que trata de la cacería ilegal que se produce en la Reserva de Vida Silvestre Amazónica Manuripi, Bolivia, durante el período de recolección de castaña, incluyendo una descripción de la regulación existente para impedir la cacería ilegal. En la cuarta sección se resume la evidencia empí- rica referida a los posibles factores que motivarían el incumplimiento de la prohibición de caza por parte de los recolectores de castaña. Considerando en conjunto la evidencia sobre incumplimiento y el enfoque teórico sobre sus posibles causas, en la quinta sección se discuten opciones de gestión para evitar el incumplimiento de la prohibición durante el periodo de recolección de castaña.

\section{¿Qué factores motivan el incumplimiento a normas que regulan el acceso a los recursos naturales?}

Becker (1968) fue el primero en desarrollar formalmente un modelo teórico en torno al comportamiento criminal de las personas, desde una perspectiva económica. Este autor señala que las decisiones de aquellos que transgreden las leyes y reglas, al igual que del resto de los individuos, responden a sus intenciones de maximizar su beneficio económico neto, esto es incluyendo ganancias por transgresión como también costos esperados por imposición de una sanción en caso de ser detectado. Bajo este modelo conceptual, los individuos cometerán un crimen siempre que el beneficio neto de cometerlo supere el beneficio esperado que se obtendría cumpliendo la ley.

A partir del planteamiento de Becker y el desarrollo de estudios que han extendido su modelo teórico, se encuentra bien establecido en la literatura un "modelo básico de disuasión" en el que la probabilidad de detección y monto de la sanción son los principales determinantes del cumplimiento con una normativa. Bajo este enfoque, los esfuerzos de fiscalización orientados a aumentar la probabilidad de detección y el recrudecimiento de las sanciones serían los principales mecanismos de política para mejorar los niveles de cumplimiento con la regulación. Si bien la probabilidad de detección suele ser baja cuando los esfuerzos de fiscalización son bastante costosos, ello debería ser contrarrestado con sanciones severas o sanciones económicas altas. Las multas deben ser los suficientemente altas como para superar los beneficios ilegales asociados al incumplimiento de la regulación. Existen varios estudios empíricos realizados para actividades pesqueras que han sugerido de manera robusta que las probabilidades de detección y sanción esperadas tienen ciertamente un efecto positivo en disuadir las violaciones (Furlong 1991; Hatcher y Gordon 2005; Viteri y Chávez 2007; Eggert y Lokina 2010).

Sutinen y Kuperan (1999) señalan que existe otro tipo de motivaciones, además de la probabilidad de detección y sanción, que afectan las decisiones de cumplimiento con una normativa y están asociadas a factores sociales y psico-sociales un tanto similares a los que discute Eliason (2003) en la denominada Teoría de Neutralización. Estos autores señalan que el desarrollo moral, la influencia social y la percepción sobre la legitimidad de la regulación y de la autoridad fiscalizadora son factores que afectan el comportamiento de los individuos con respecto a una norma. En este sentido, Sutinen y Kuperan (1999) proponen una extensión al modelo de disuasión de Becker en el que incluyen motivaciones intrínsecas y extrínsecas, adicionalmente a las económicas.

Los factores del desarrollo moral están asociados a una motivación intrínseca y se refieren a los valores personales que influyen en la decisión de cumplimiento. Sutinen y Kuperan (1999), señalan que el sentido de moralidad obliga a los individuos a comportarse de manera correcta y por tanto podría motivar a cumplir con una regulación a pesar de los beneficios económicos potenciales asociados a su incumplimiento. De acuerdo a estos autores, la presencia de motivaciones intrínsecas reduce el riesgo de infracción a la norma cuando esta transgresión está en contra de los valores morales del individuo.

La influencia social y la legitimidad están relacionadas con factores extrínsecos de motivación. La primera considera que la presión del entorno social tiene un efecto importante sobre la decisión individual. Se puede identificar el comportamiento y la opinión de los pares como los factores que influyen en las decisiones de los individuos sobre el cumplimiento con la norma. La hipótesis sugeriría que, en presencia de influencia social un individuo es más pro- 
penso a cumplir con la normativa mientras mayor cumplimiento perciba entre sus pares (Geerken y Gove 1975; Vogel 1974; Witte y Woodbury 1985. Citados en Sutinen y Kuperan 1999). La legitimidad incluye características sobre la creación y aplicación de la regulación, además de la percepción sobre la autoridad encargada de aplicar la norma. Un factor determinante de la legitimidad es la justicia con la que se imparten los procedimientos de fiscalización. También se ha sugerido que regímenes de co-gestión, donde los regulados desempeñan un papel importante en la toma de decisiones y en el proceso de elaboración y aplicación de la norma, pueden ser beneficiosos para mejorar el cumplimiento (Eggert y Ellergård 2003). La hipótesis en torno a la legitimidad sugiere que mientras más legítimas sean percibidas la autoridad y la regulación, existirá mayor propensión al cumplimiento de parte de los individuos.

Existe una cantidad extensa de estudios en regulación pesquera que han aportado evidencia consistente respecto a que el desarrollo moral, la influencia social y la legitimidad de la regulación y autoridad fiscalizadora son factores determinantes del cumplimiento a una normativa (Kuperan y Sutinen 1998; Hatcher et al. 2000; Nielsen y Mathiessen 2003; Hatcher y Gordon 2005; Viteri y Chávez 2007; Eggert y Lokina 2010; Dresdner et al. (datos sin publicar)).

En resumen, existe un modelo de disuasión ampliado que entrega una serie de hipótesis sobre los factores que podrían estar determinando el incumplimiento de los agentes a regulaciones que pretenden conservar los recursos naturales. Este modelo será aplicado en una sección siguiente al análisis de la cacería ilegal.

\section{El caso de la Reserva Manuripi: Cacería ilegal en el periodo de recolección de castaña}

La Reserva Nacional de Vida Silvestre Amazónica Manuripi es un área protegida ubicada al norte de Bolivia, en el departamento de Pando (Fig. 1). Una de las principales razones de su creación fue la protección de la fauna silvestre que alberga. La recolección de castaña (Bertholletia excelsa) es la principal actividad económica para las familias que viven en la Reserva y para aquellos recolectores que normalmente viven fuera del área protegida y son contratados para trabajar temporalmente en predios privados. En el año 2010, Manuripi aportó aproximadamente el $12 \%$ de la producción nacional de castaña (Elías 2010).

La Reserva Manuripi tiene un área aproximada de 747000 ha. y en su interior habitan cerca de 1700 personas distribuidas en 10 comunidades y 36 predios privados (barracas). Conjuntamente, ambos tipos de propiedad (comunal y privada) ocupan el $25 \%$ del área protegida, mientras que la superficie restante corresponde a tierras fiscales o de propiedad estatal. Si bien en las comunidades, el régimen de propiedad es común, cada una hace la distribución interna de la tierra por lo que cada familia dispone de una parcela de terreno, haciéndose, de esta forma, independientes en sus labores agrícolas y de extracción de castaña. Cada predio familiar tiene en promedio 500 ha. y su economía se caracteriza por ser un sistema mixto que combina la agricultura y la ganadería con actividades extractivas de castaña, goma y otros. La caza y pesca son actividades complementarias (SERNAP 2011b).

Las propiedades privadas mantienen poblaciones de familias de trabajadores para labores de mantenimiento y a cambio de ello el propietario les permite una agricultura para consumo, caza y pesca en sus predios. Estas familias representan una población aproximada de 250 personas. La extensión de los predios privados oscila entre 500 y 2000 ha., dependiendo del tamaño familiar. Durante el periodo de recolección, los propietarios pueden acceder a áreas adicionales de bosque que corresponden a tierras fiscales, lo cual se denomina "derecho expectaticio". Estas áreas tienen extensiones que varian entre 400 y 15000 ha. por predio y deben ser usadas únicamente para el aprovechamiento de castaña. $\mathrm{El}$ manejo de mayores áreas de bosque genera la contratación de recolectores temporales que provienen de fuera de la Reserva, en un número que supera hasta en diez veces la población de trabajadores estables (SERNAP 2011b). Esta característica del trabajo

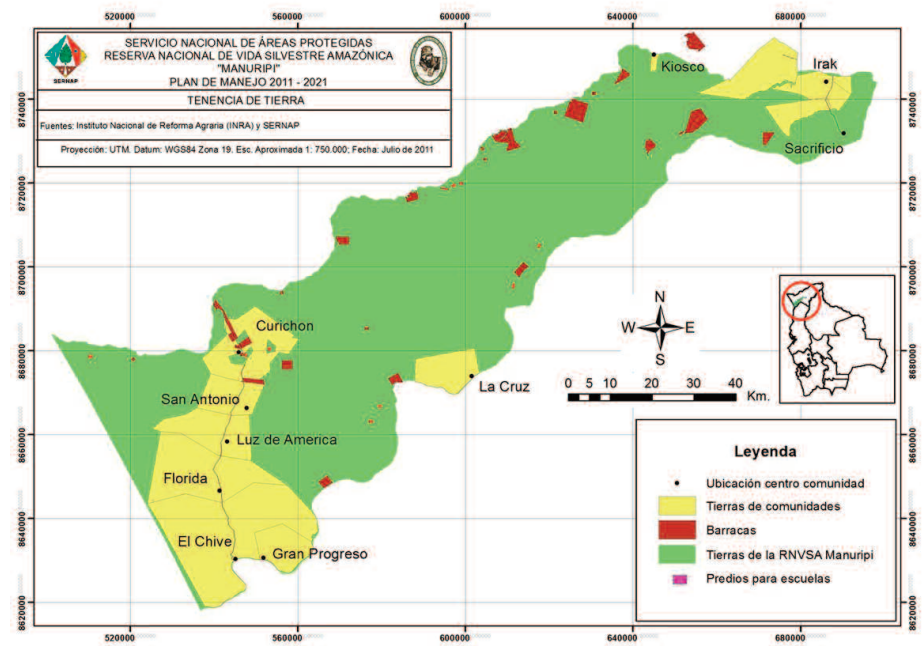

Figura 1. Mapa de la zona de estudio, la Reserva Nacional de Vida Silvestre Amazónica Manuripi (Bolivia). Fuente: SERNAP (Servicio Nacional de Áreas Protegidas, Bolivia)

en predios privados es similar en toda el área de aprovechamiento de castaña en Bolivia.

A pesar de su importancia y la característica no maderable de su aprovechamiento, la extracción de castaña está asociada a problemas ambientales como la cacería excesiva por parte de los recolectores que permanecen internados en el bosque durante el periodo de recolección (SERNAP 2011b). En la Reserva Manuripi existen 150 especies de mamíferos, 501 especies de aves, 77 especies de reptiles, 83 especies de anfibios y 112 especies de peces (HERENCIA 2003). La fauna cinegética corresponde a 53 especies de mamíferos, 35 especies de aves y una de reptil (Rechberger 2010). Estas especies son aquellas sobre las cuales existe presión por cacería, tanto en comunidades como en predios privados. Estudios biológicos de monitoreo de la fauna de la Reserva muestran que la caza desarrollada durante la recolección de castaña (incluyendo zonas aplicadas al derecho expectaticio) genera una presión considerable en al menos cuatro especies de mamíferos y tres de aves, las cuales se vieron afectadas en abundancia poblacional (Rechberger 2008 y 2010).

Durante el tiempo de permanencia al interior del bosque, la cacería ha sido una actividad comúnmente desarrollada por los recolectores de castaña, como forma barata de aprovisionamiento de carne y debido al insuficiente suministro de carne no silvestre en los predios privados. Considerando que en el periodo de cosecha son miles los recolectores que se trasladan a la selva, en su mayoría acompañados por sus familias (20 000 familias aproximadamente en toda la Amazonía boliviana), la caza se constituye en una amenaza para la conservación de la vida silvestre. Una investigación sobre la caza asociada a la recolección de castaña en Bolivia identifica que dos de las especies preferidas por los cazadores ( Tapirus terrestres y Ateles chamek) son escasas durante el período de la cosecha debido a su sobreexplotación (Rumiz y Maglianesi 2001, citado en Townsend et al. 2002).

Considerando el potencial impacto negativo de la caza en la conservación de la Reserva Manuripi, además de las preocupaciones sociales sobre las condiciones de trabajo de los recolectores dentro el bosque, la Dirección del área protegida implementa desde el año 2008 un reglamento para controlar las actividades en torno a la recolección de castaña, incluyendo la cacería. Este reglamento fue consensuado entre comunidades, propietarios privados y la administración de la Reserva. Dentro esta normativa se destaca la diferenciación entre los derechos de caza para pobladores locales y recolectores contratados procedentes de fuera del área protegida. Mientras que las comunidades y los propietarios de tierras privadas tienen el derecho a la caza para consumo familiar, los recolectores contratados están prohibidos de cazar aún con fines de consumo, (Bolivia 2008). 
El proceso de fiscalización en torno a la regulación de la castaña en la Reserva Manuripi es llevado a cabo por el SERNAP (Servicio Nacional de Áreas Protegidas) a través del personal administrativo del área protegida. En el reglamento se define un límite para el número de recolectores en cada propiedad privada de acuerdo al tamaño de su zona de aprovechamiento. Los propietarios privados deben cumplir con este requisito a fin de ser autorizados para realizar la recolección de castaña en sus predios. El proceso de monitoreo establece visitas e inspecciones sorpresivas durante el período de recolección, las cuales se centran en las viviendas temporales al interior del bosque, donde los recolectores y sus familias permanecen durante casi toda la temporada de cosecha. Los recolectores también son inspeccionados antes de entrar a la Reserva y en las instalaciones de los predios privados. Con respecto al cumplimiento con la prohibición de cacería, las inspecciones se centran en controlar la posesión de armas y municiones, verificar la existencia de remanentes de carne o restos de animales silvestres en las viviendas y evidenciar la existencia de animales cazados vivos y mantenidos en captura.

Desde su implementación en 2008 , se han detectado significativos niveles de incumplimiento al reglamento en general y a la prohibición de cacería en particular. Informes de monitoreo señalan violaciones considerables a la prohibición de caza en predios privados. En 2008 , el $50 \%$ de las propiedades privadas cometió infracciones. Se detectaron armas de fuego en $44 \%$ de las propiedades privadas inspeccionadas, restos de animales salvajes en el $25 \%$ de las propiedades y animales capturados vivos en $13 \%$ de ellas. En 2009 , el $66 \%$ de las propiedades privadas inspeccionadas reporta transgresiones. El personal del área protegida encontró armas de fuego en $51 \%$ de ellas, vestigios de animales cazados en $24 \%$ y animales capturados vivos en un $16 \%$ de los predios inspeccionados. El informe de monitoreo de 2011 muestra que el $100 \%$ de las propiedades privadas viola el reglamento de caza en algún aspecto. Se detectó el uso de armas de fuego en $72 \%$ de las propiedades inspeccionadas; el $100 \%$ de éstas evidenció el uso de municiones y restos de animales salvajes, mientras que un $44 \%$ presentó animales capturados con vida (Ver Tabla 1) (SERNAP 2008, 2009, 2011a)

Aunque no se cuenta con información precisa sobre el nivel de caza en propiedades privadas de la Reserva, los informes de monitoreo a la fauna y de cumplimiento a la regulación ponen en evidencia el desarrollo de la cacería a pesar de la prohibición. De esta manera, esta actividad ilegal aún representa una potencial amenaza para la conservación de la fauna de la Reserva Manuripi y dado que la protección de la vida silvestre fue una de las principales razones para su creación, la Autoridad Ambiental está preocupada por reducir la caza ilegal durante la temporada de cosecha de castaña. Surge entonces la pregunta sobre cuáles son los factores que influyen en las decisiones de cacería de los recolectores y si estos factores están relacionados únicamente a eventuales ahorros por gasto en alimentación, los esfuerzos de fiscalización de la autoridad ambiental (vigilancia destinada a detectar transgresiones y aplica- ción de sanciones en caso de detección) o existirán además otros determinantes del incumplimiento de los recolectores a la prohibición de cacería, incluyendo por ejemplo factores sociales o culturales. Este conocimiento es relevante desde la perspectiva del diseño de políticas y acciones que ayuden a alcanzar los objetivos de conservación del reglamento.

\section{Determinantes de la cacería durante la zafra de castaña en la Reserva Manuripi}

En esta sección aplicamos los modelos de disuasión presentados en la segunda sección, al análisis de los determinantes del incumplimiento a la prohibición de caza en el caso de estudio analizado en este artículo. Las afirmaciones y discusión se basan en fuentes secundarias de información, así como en entrevistas realizadas por los autores a representantes del personal de protección de la Reserva, dueños de predios privados y recolectores de castaña entre los años 2009 y 2010. Adicionalmente, el análisis se basa en los resultados de Espinoza (2013), datos sin publicar, que estudia los determinantes del incumplimiento a la prohibición de cacería en la Reserva Manuripi.

Aplicando el modelo de disuasión simple a la explicación del comportamiento delictivo de las personas (Becker 1968), podríamos esperar que el nivel de cumplimiento con la prohibición de caza para la Reserva Manuripi sea reducido, tanto por una baja probabilidad de detección como por las sanciones aplicadas. Con respecto a la probabilidad de detección, y en base a fuentes secundarias de información, así como a las entrevistas realizadas a los diferentes actores, identificamos varios factores que nos dan indicios de que existiría una probabilidad muy baja:

Dimensiones y características geográficas del área protegida. La extensión considerable de la Reserva y su delimitación natural comprendida por dos grandes ríos, la hacen vulnerable a la evasión de control de ingreso al área.

Personal de protección y de monitoreo reducidos. La Reserva Manuripi fue asignada con un cuerpo de protección conformado por 13 guardaparques y un jefe de protección (SERNAP 2011b). Durante la etapa de recolección de castaña se suman de 3 a 5 personas que realizan el monitoreo al cumplimiento del Reglamento en predios privados y comunidades de la Reserva. Este personal resulta insuficiente para garantizar un control adecuado de los cerca de 900 recolectores contratados que ingresan al área protegida, en su mayoría con familias.

Condiciones de accesibilidad dentro el área protegida. El clima Iluvioso que acompaña la temporada de recolección de castaña provoca malas condiciones de los caminos y senderos dentro el bosque, lo cual obstaculiza las inspecciones a las viviendas temporales de los recolectores. Estudios como Holmern et al. (2007) confirman que la estación lluviosa está asociada a menores probabilidades de detección.

Tabla 1. Resultados del proceso de monitoreo a la prohibición de cacería según reportes anuales del SERNAP (Servicio Nacional de Áreas Protegidas, Bolivia). Fuente: reportes de monitoreo del SERNAP.

\begin{tabular}{|c|c|c|c|c|c|c|c|c|}
\hline \multirow[t]{2}{*}{ Año } & \multirow[t]{2}{*}{$\begin{array}{l}\text { Número } \\
\text { de recolectores }\end{array}$} & \multirow[t]{2}{*}{$\begin{array}{l}\text { Recolectores } \\
\text { inspeccionados }\end{array}$} & \multirow[t]{2}{*}{$\begin{array}{c}\text { Predios } \\
\text { inspeccionados }\end{array}$} & \multirow[t]{2}{*}{$\begin{array}{c}\text { Número } \\
\text { de inspecciones }\end{array}$} & \multicolumn{3}{|c|}{$\begin{array}{l}\text { Infracciones a la cacería } \\
\text { en predios privados }\end{array}$} & \multirow[t]{2}{*}{$\begin{array}{l}\text { Sanciones } \\
\text { aplicadas }\end{array}$} \\
\hline & & & & & Armas & Caza & Capturas & \\
\hline 2008 & 848 & 506 & 32 & 1 & $44 \%$ & $25 \%$ & $13 \%$ & $\begin{array}{l}\text { Decomiso } \\
\text { preventivo de } \\
\text { armas }\end{array}$ \\
\hline 2009 & 972 & 720 & 37 & 1 & $51 \%$ & $24 \%$ & $16 \%$ & $\begin{array}{l}\text { Decomiso de } \\
\text { armas }\end{array}$ \\
\hline 2011 & 856 & 255 (aprox.) & 25 & 1 & $72 \%$ & $100 \%$ & $44 \%$ & $\begin{array}{l}\text { Decomiso de } \\
\text { armas }\end{array}$ \\
\hline
\end{tabular}

1: Se consideró el número total de recolectores autorizados cada año. 2: Se consideró la relación entre el número de predios privados que cometieron infracciones y el número total de predios privados inspeccionados cada año. 
Fácil evasión de control e ingreso de armas. Son pocos los puntos de control de ingreso a la Reserva y por ellos llegan masivamente los recolectores que trabajarán no sólo en Manuripi sino en otros predios privados de la región. De esta forma, la evasión de control puede ser considerable y ello se constata con la detección de armas al interior de los predios privados, reportada en los informes de monitoreo.

La cacería también se puede realizar sin el uso de armas: Existen recolectores que afirman que algunas especies de animales son cazadas con palos o con los machetes que utilizan en el trabajo de quebrado de los frutos de la castaña (Espinoza 2013, datos sin publicar). Esto significa que no sólo aquellos que tienen armas de fuego son propensos a cazar.

Reducido número de inspecciones durante el proceso de monitoreo. De acuerdo a los informes de monitoreo para los años 2008, 2009 y 2011, las viviendas temporales de los recolectores fueron inspeccionadas en su mayoría una única vez. Considerando que la temporada de recolección de castaña dura aproximadamente 90 días, los recolectores pueden haber cazado en varias ocasiones sin haber sido detectados.

Fácil encubrimiento de la cacería. Los restos de los animales cazados y las armas mismas pueden ser encubiertos con facilidad en el bosque ante el conocimiento de la llegada del personal de monitoreo.

Para nuestro caso particular de análisis, adicional a una probabilidad de detección baja, existen sanciones poco severas y poca claridad en relación a la definición y aplicación de multas por cacería. Estos dos elementos en conjunto sugerirían altos niveles de incumplimiento con la regulación en la Reserva Manuripi. Las sanciones por incumplimiento al Reglamento de la castaña (Bolivia 2008) sólo se remiten a normas nacionales tales como el Reglamento General de Áreas Protegidas, (Bolivia 1997), donde se señala que las sanciones administrativas se establecen de acuerdo a la gravedad de la infracción, pero no se hace una diferenciación entre los distintos tipos o grados de infracción. De manera general se establece que ante contravenciones a disposiciones legales que conciernen a las áreas protegidas se aplicarían multas que oscilan entre 1 y 300 "días multa", donde un "día multa" equivale al $30 \%$ del salario mínimo nacional. Esto significa que las multas oscilarían entre 43 a 12930 dólares. (Se consideró el salario mínimo mensual de Bolivia para el año 2012 y el tipo de cambio 6,96 bolivianos = 1 \$US).

Ni el Reglamento para la Recolección de Castaña ni el Reglamento General de Áreas Protegidas establecen multas específicas para las actividades de caza ilegal. Adicionalmente, en la regulación para la recolección de castaña existe ambigüedad en torno al sujeto sobre el cual recaería la sanción económica en caso de aplicarse (Espinoza 2013, datos sin publicar). No está claro si debería ser el dueño del predio privado el que cargaría con la multa, al ser éste responsable por sus trabajadores contratados y por asegurarse de que éstos conozcan y acaten el reglamento, o si la multa se aplicaría directamente al recolector infractor. El decomiso de armas a recolectores contratados es la única medida señalada en los reportes de monitoreo como sanción, tanto por portar el arma ilegalmente como por cacería.

Desde la implementación de la regulación de castaña en la Reserva Manuripi en 2008, los esfuerzos de fiscalización se han enfrentado a otros aspectos de tipo institucional y administrativos que dificultaron los procesos de monitoreo. Los informes anuales muestran que en la práctica, cada monitoreo fue diferente debido a que no se ha logrado definir un proceso uniforme para ser implementado. La duración de cada uno, el personal involucrado y el número y calidad de las inspecciones fueron variables. Ello también estuvo relacionado a la inestabilidad institucional que existió al interior del SERNAP y la Dirección de la Reserva Manuripi, ya que en el período 2008-2011 hubo tres directores para el área protegida, los cuales determinaron las diferentes actividades y el alcance de cada proceso de monitoreo que a su vez estuvieron sujetos a la disponibilidad de recursos económicos anuales. Esta información fue ve- rificada a través de las entrevistas realizadas a los directores del área protegida.

En 2008 y 2009 se inspeccionó casi el $90 \%$ de las viviendas temporales de recolectores en tierras privadas. En 2010, el proceso de seguimiento fue breve y tardío por lo que su alcance fue bastante reducido. En 2011, se definió una muestra de las viviendas a ser inspeccionadas, abarcando un 30\% del total (Espinoza 2013, datos sin publicar). Los alcances de cada uno de los procesos de monitoreo, además de las infracciones detectadas en cacería figuran en la Tabla 1. Como se podrá observar, el número de recolectores y predios inspeccionados cada año fueron variables. El decomiso de armas de fuego a recolectores infractores se aplicó únicamente en los años 2009 y 2011 e incluso para esos años los informes indican que hubo problemas logísticos para incautar varias armas. No se conoce información acerca de la aplicación de multas a recolectores que fueron detectados violando la prohibición de caza.

El modelo de disuasión ampliado (Sutinen y Kuperan 1999) incluye el análisis de factores como el desarrollo moral, la influencia social, y la legitimidad, como determinantes adicionales del cumplimiento de una normativa. Para nuestro caso particular de análisis, si bien el desarrollo moral sería difícil de evaluar sin conocer y estudiar mejor a los recolectores de castaña, existen algunos indicios que nos llevan a creer que la influencia social y la legitimidad de la regulación pueden tener un efecto en el nivel de cumplimiento con la prohibición de caza. Por un lado, los recolectores comparten viviendas entre varias familias dentro el bosque y ello puede sugerir que su comportamiento en torno a la norma se puede ver influenciado por otros recolectores. A partir de la información proporcionada por guardaparques y las encuestas a los recolectores, se conoce que se acostumbra compartir algunos productos como alimentos durante el periodo de recolección, pero también se suele compartir las armas de fuego. Esto significa que aquellos que logran introducir sus armas dentro la Reserva pueden influenciar a otros recolectores para cazar. Espinoza (2013, datos sin publicar) identifica que aquellos recolectores que perciben que sus compañeros cazan, son más propensos comportarse de la misma forma.

Por otro lado, si bien no se tiene certeza de la percepción que los recolectores de castaña podrían tener sobre la autoridad fiscalizadora, en este caso la Dirección del Área Protegida, se conoce que la Regulación para la Recolección de Castaña fue aprobada sin tomar en cuenta la opinión de los recolectores contratados, por ser actores externos a la Reserva (Entrevistas a personal del Servicio Nacional de Áreas Protegidas). Es decir que los recolectores tuvieron una participación pasiva en la elaboración de la norma. Este factor puede tener un efecto negativo en la percepción sobre la legitimidad de la regulación. Es probable también que la diferenciación entre los derechos de cacería que tienen los actores locales frente a la prohibición impuesta a los recolectores contratados pueda ser desfavorable para la legitimidad de la prohibición de cacería en particular. Otros factores adicionales como la ausencia de imposición de multas y sanciones poco estrictas pueden también dañar la imagen de la Dirección del Área Protegida y su credibilidad como autoridad fiscalizadora.

Adicionalmente a los factores analizados, creemos que el caso de los recolectores de castaña puede implicar otros aspectos que afecten su comportamiento hacia la prohibición de caza. Específicamente, consideramos que algunos factores culturales y el nivel de conocimiento de la regulación pueden ser determinantes del cumplimiento con el reglamento. Los factores culturales relacionados a la costumbre por cazar y preferencias por el consumo de carne silvestre que existe entre las comunidades y población amazónica podrían afectar los niveles de cacería ilegal. Schenck et al. (2006) observan que la preferencia por la carne silvestre frente a otras alternativas puede incidir en las decisiones de cacería. Para el caso particular de la Reserva Manuripi, Espinoza (2013, datos sin publicar) encontró que los recolectores que acostumbran consumir carne de monte fuera de la época de recolección de castaña son más propensos a infringir la regulación y cazar. 
El nivel de conocimiento de la regulación se relaciona a la información que la población regulada tiene sobre la normativa. En el caso de los recolectores de castaña, dado que los individuos contratados año a año para trabajar en los predios privados pueden ser diferentes y probablemente habrá muchos que van por primera vez a la Reserva Manuripi, es probable que el nivel de conocimiento del Reglamento para la Recolección de Castaña, incluida la prohibición de cacería, sea variable y en algunos casos escaso. Más aún si se considera que la norma no ha estado vigente por muchos años. Winter y May (2001) y Alayon (2011) encontraron que el conocimiento de las reglas es un factor determinante para el cumplimiento.

Finalmente, considerando que la cacería durante el periodo de recolección de castaña es desarrollada principalmente con fines de consumo (Espinoza 2013, datos sin publicar), creemos que la disponibilidad y accesibilidad a carnes substitutas a la de monte en los predios privados, así como los costos que estén asociadas a éstas también pueden constituirse en factores relevantes para las decisiones de cacería de los recolectores contratados. Si bien los propietarios de los predios privados están obligados de proveer carne no silvestre a sus trabajadores, lo cual también está estipulado en el Reglamento para la Recolección de Castaña, los informes de monitoreo reflejan que existe un nivel significativo de incumplimiento en este aspecto de la norma. Espinoza (2013, datos sin publicar) evidencia que el abastecimiento de carne en los predios privados de la Reserva es uno de los factores más relevantes para el cumplimiento con la prohibición de cacería. Sin embargo, dado que en ese estudio también se identificó la existencia de recolectores que cazan con fines comerciales, se observó que para este grupo la disponibilidad de alternativas substitutas a la carne silvestre no es relevante en sus decisiones de cacería.

\section{¿Cuáles son las medidas necesarias para disminuir el nivel de incumplimiento con la prohibición de cacería?}

Sobre la base de los determinantes del cumplimiento identificados en las secciones anteriores, tanto aquellos que la teoría en torno a los modelos de disuasión señala, como los probados por el estudio de Espinoza (2013, datos sin publicar), en esta sección discutimos sobre las medidas que se podrían adoptar para reducir la cacería ilegal en la Reserva Manuripi.

Si se confirma que los factores de disuasión son determinantes del cumplimiento de la prohibición de cacería, las acciones necesarias implicarían mejorar los esfuerzos de monitoreo, control, detección y sanción de los recolectores que cazan. Si bien los costos de fiscalización son altos, la claridad en cuanto al monto de las sanciones económicas y su efectiva aplicación debería ser revisada y mejorada por la autoridad ambiental. Tal como está definida actualmente la sanción monetaria, implica niveles extremos que la llevan a ser muy pequeña o demasiado grande. Este tipo de sanción no resulta ser muy informativa para la decisión de cumplir o no con la norma. Además, si la sanción es demasiado elevada puede ser considerada como poco probable de ser efectivamente aplicada Adicionalmente, el proceso administrativo relacionado a la imposición de multas debería también ser lo más claro y menos burocrático posible. De mantenerse una definición poco clara en las multas y el proceso administrativo para su aplicación, se tendería a reducir aún más el poder disuasivo de las actividades de fiscalización.

Consideramos que los factores en torno a la influencia social y la moralidad, además de factores culturales como la costumbre por el consumo de la carne silvestre considerado en Espinoza (2013, datos sin publicar), podrían ser afectados pero no en el corto plazo y de acuerdo a las acciones dirigidas a modificarlos y a la evolución de las costumbres de la población. La autoridad ambiental podría mejorar y aumentar los procesos de información y sensibilización acerca de la regulación y las consecuencias del exceso de caza con el fin de crear conciencia entre los recolectores contratados sobre el problema de la cacería en la conservación de la Reserva
Manuripi. Sin embargo, los cambios en los hábitos culturales, así como los valores morales dependerían también de los procesos educativos, así como de la evolución de las características de la población de recolectores de castaña.

Para mejorar la percepción de legitimidad de la regulación y de la autoridad ambiental, sería necesario motivar a los recolectores a cumplir la regulación de tal forma que la asocien con beneficios y no sólo con costos y perjuicios para ellos. Socializar la regulación para enfatizar que la norma también pretende mejorar las condiciones de trabajo y alimentación de los recolectores de castaña puede ayudar a cambiar favorablemente la percepción hacia el reglamento en su conjunto. Esto significa que las acciones también deberían enfocarse en mejorar el control de la oferta de carne no silvestre y sus precios en las propiedades privadas. Esta última medida es necesaria dado que existe ya una referencia que señala que el abastecimiento de substitutos de carne silvestre en los predios privados de la Reserva podría cambiar el comportamiento delictivo de los recolectores que cazan con fines de consumo.

Finalmente, el conocimiento sobre la regulación y la existencia de la prohibición de caza como factores relevantes del cumplimiento con el reglamento, implicarían que los esfuerzos se enfoquen en un mayor trabajo de divulgación y socialización de la normativa entre los recolectores contratados.

En suma, existen varias medidas que se pueden implementar para reducir el incumplimiento a la normativa sobre prohibición de cacería en la Reserva Manuripi. Sin embargo, no existe la certeza de que todas funcionen en la práctica. Un proceso de implementación de distintas medidas de prueba y error puede entregar mayor evidencia sobre cuáles son las más efectivas. Lo que no parece razonable es concentrarse únicamente en medidas que apunten a aumentar los castigos y la probabilidad de ser detectado. Proponemos que en principio se ponga atención a aquellos factores que ya fueron probados como relevantes para el caso de la Reserva Manuripi, que según el estudio de Espinoza (2013, datos sin publicar) son el abastecimiento de carne no silvestre en los predios privados, el hábito por el consumo de la carne silvestre y la influencia de los recolectores que cazan sobre otros que probablemente no cazarían en caso de percibir cumplimiento entre sus pares. Para considerar seriamente los otros potenciales determinantes de la cacería ilegal, serán necesarios mayores estudios que puedan guiar y definir más claramente las acciones de la autoridad ambiental competente.

A partir de la revisión de literatura sobre cumplimiento y cacería desarrollados en este artículo, logramos identificar los posibles determinantes del cumplimiento para una normativa específica sobre prohibición de cacería. La información proporcionada puede guiar las acciones y esfuerzos de fiscalización de las autoridades encargadas de aplicar la norma. Sin embargo, para ello se hace necesario profundizar en la investigación sobre los factores que afectan el cumplimiento de la prohibición de cacería en la Reserva Manuripi. Observamos que, dependiendo de cuáles sean los factores relevantes, las implicancias de gestión serán diferentes.

\section{Agradecimientos}

Agradecemos los detallados y útiles comentarios de los dos Revisores anónimos de Ecosistemas y de Lucía Gálvez, Editora invitada de la Revista. Espinoza reconoce y agradece el apoyo financiero para esta investigación recibido del Programa de Economía Ambiental para América Latina y el Caribe (LACEEP) y el apoyo técnico de Conservación Estratégica Bolivia. Dresdner y Chávez reconocen y agradecen el financiamiento parcial recibido a través del proyecto NS 100007, de la Iniciativa Científica Milenio, del Ministerio de Economía, Fomento y Turismo del Gobierno de Chile. Finalmente, un agradecimiento especial a la Dirección de la Reserva Manuripi y a su Cuerpo de Protección por el acompañamiento en los viajes de campo y por facilitar información de mucha utilidad para este artículo. 


\section{Referencias}

Alayon, L. 2011. Regulatory Compliance in small-scale fisheries on San Andres and Old Providence Islands (Colombia). LACEEP. Working Paper Series WP29. Costa Rica.

Bassett, T. 2005. Card-carrying hunters, rural poverty, and wildlife decline in northern Côte d'Ivoire. The Geographical Journal 171:24-35

Becker, G.S. 1968.Crime and Punishment: An Economic Approach. Journal of Political economy 76:169-217.

Bolivia 1997. Decreto Supremo N²4781 del 31 de julio de 1997. Reglamento General de Áreas Protegidas. Gaceta Oficial del Estado. Compendio: Medio Ambiente. Edición 2025 de 01 de agosto de 1997, 43 pp.

Bolivia 2008. Resolución Administrativa-DE-N.044/2008 del 19 de diciembre de 2008. Reglamento para el Control de las Actividades de la Zafra en la Reserva Nacional de Vida Silvestre Amazónica Manuripi. SERNAP. La Paz, Bolivia.

Brashares, J.S., Golden, C.D., Weinbaum, K.Z., Barrett, C.B., Okello, G.V. 2011. Economic and geographic drivers of wildlife consumption in rural Africa. Cornell University, Ithaca, Estados Unidos.

Eggert, H., Ellergård, A. 2003. Fishery control and regulation compliance: a case for Co-management in Swedish commercial fisheries. Marine Policy 27:525-533.

Eggert, H., Lokina, A. 2010. Regulatory Compliance in Lake Victoria Fisheries. Environment and Development Economics 15:197-217

Elías, B. 2010. Diagnóstico de los Sistemas de Producción en la Reserva Manuripi. Elaborado para el Servicio Nacional de Áreas Protegidas. La Paz, Bolivia.

Eliason, S.L. 2003. Illegal Hunting and Angling: The Neutralization of Wildlife Law Violations. Society and Animals 11:3

Furlong, W. 1991. The Deterrent Effect of Regulatory Enforcement in the Fishery. Land Economics 67:116-129.

Geerken, M., Gove, W.R. 1975. Deterrence: some theoretical considerations. Law and Society Review 9:495-513

Hatcher, A., Jaffry, S., Thébaud, O., Bennett, E. 2000. Normative and Social influences Affecting Compliance with Fishery Regulations. Land Economics 76:448-461.

Hatcher, A., Gordon, D. 2005. Further Investigations into the Factors Affecting Compliance with U.K. Fishing Quotas. Land Economics 81:71-86.

HERENCIA 2003. Biodiversidad de la Reserva Nacional de Vida Silvestre Amazónica Manuripi. Cobija, Bolivia.

Holmern T., Muya, J., Røskaft, E. 2007. Local law enforcement and illegal bushmeat hunting outside the Serengeti National Park, Tanzania. Environmental Conservation 34(1):55-63

Johannesen, A.B. 2005. Wildlife conservation policies and incentives to hunt: an empirical analysis of illegal hunting in western Serengeti, Tanzania. Environment and Development Economics 10:271-292

Kuperan K., Sutinen J. 1998. Blue water crime: deterrence, legitimacy, and compliance in fisheries. Law and Society Review 32:309-338

Loibooki M., Hofer, H., Campbell, K., East, M. 2002. Bushmeat hunting by communities adjacent to the Serengeti National Park, Tanzania: the importance of livestock ownership and alternative sources of protein and income. Environmental Conservation 29:391-398.

Nielsen, J.R., Mathiesen, C. 2003. Important factors influencing rule compliance in fisheries lessons from Denmark. Marine Policy 27:409-416.
Nielsen, M.R. 2006. Importance, cause and effect of bushmeat hunting in the Udzungwa Mountains, Tanzania: implications for community based wildlife management. Biological conservation 128:509-516

Rumiz, D.I., Maglianesi, M.A. 2001. Hunting impacts associated to Brazil nut extraction in the Bolivian Amazon. Vida Silvestre Neotropical 10:1929.

Rechberger, J. 2008. Monitoreo de la fauna cinegética en la Reserva Nacional de Vida Silvestre Amazónica Manuripi. Elaborado para el Servicio Nacional de Áreas Protegidas. La Paz, Bolivia.

Rechberger, J. 2010. Monitoreo de la fauna cinegética en la Reserva Nacional de Vida Silvestre Amazónica Manuripi. Elaborado para el Servicio Nacional de Áreas Protegidas. La Paz, Bolivia.

Schenck, M., Effa, E. N., Starkey, M., Wilkie, D., Abernethy, K., Telfer, P., Godoy R., Treves, A. 2006. Why People Eat Bushmeat: Results From Two-Choice, Taste Tests in Gabon, Central Africa. Human Ecology 34:3

SERNAP 2008. Informe del monitoreo a la zafra de castaña 2007-2008 en la Reserva Nacional de Vida Silvestre Amazónica Manuripi. La Paz, Bolivia.

SERNAP 2009. Informe del monitoreo a la zafra de castaña 2008-2009 en la Reserva Nacional de Vida Silvestre Amazónica Manuripi. La Paz, Bolivia.

SERNAP 2011a. Informe del monitoreo a la zafra de castaña 2010-2011 en la Reserva Nacional de Vida Silvestre Amazónica Manuripi. La Paz, Bolivia.

SERNAP 2011b. Plan de Manejo para la Reserva Nacional de Vida Silvestre Amazónica Manuripi 2011-2021. La Paz, Bolivia.

Skonhoft, A., Solstad, J.T. 1996. Wildlife management, illegal hunting and conflicts. A bioeconomic analysis. Environment and Development Economics 1:165-18

Sutinen, J.G., Kuperan, K. 1999. A Socioeconomic Theory of Regulatory Compliance in Fisheries. International Journal of Social Economics. 26:174-193

Sykes, G.M., Matza, D. 1957. Techniques of neutralization: A theory of delinquency. American Sociological Review 22:664-670.

Townsend, W.R., Rumiz, D.I., Solar, L. 2002. El riesgo de la cacería durante las operaciones Forestales: Impacto sobre la fauna silvestre en una concesión forestal en Santa Cruz. Revista Bolivia Ecológica 11:47-53.

Viteri, C., Chávez, C. 2007. Legitimacy, Local Participation, and Compliance of the Fishing Regulations in the Galapagos Marine Reserve Reservation Marina, Ocean and Coastal Management 50:253-274.

Vogel, J. 1974. Taxation and public opinion in Sweden: an interpretation of recent survey data, National Tax Journal 499-513.

Wilkie, D.S., Carpenter, J.F.1999. Bushmeat hunting in the Congo Basin: an assessment of impacts and options for mitigation. Biodiversity and Conservation 8:927-955

Willcox, A.S., Nambu D.M. 2007. Wildlife hunting practices and bushmeat dynamics of the Banyangi and Mbo people of Southwestern Cameroon. Biological Conservation 134:251-261

Winter, S., May, P. 2001. Motivation for compliance with Environmental Regulations. Journal of Policy Analysis and Management 20:675-69.

Witte, A., Woodbury, D. 1985, The effect of tax laws and tax administration on tax compliance: the case of the US individual income tax. NationalTaxJournal 1-13. 